\title{
Frame-semantische Präliminarien
}

\section{Zusammenfassung}

Die Frame-Semantik erfasst die Bedeutung sprachlicher Einheiten, indem sie die Frage stellt, welches Wissen Sprecher_innen und Rezipient_innen kognitiv zur Verfügung haben (müssen), um eine solche sprachliche Einheit (Wörter, Phrasen, Sätze, Texte) verstehen und gebrauchen zu können. Dabei vereint dieser Begriff eine Vielzahl an Forschungsansätzen, die sich teils erheblich voneinander unterscheiden. Für die Einführung des Frame-Begriffs in die linguistische Semantik zeichnet Charles Fillmore verantwortlich, der sich in seiner Konzeption der Frame-Semantik insbesondere an semantischen Rollen orientierte. Andere linguistische Ansätze orientieren sich stärker am kognitionspsychologischen Frame-Konzept. In dieser Arbeit wird ein Frame als kognitives Repräsentationsformat verstanden, das Wissen organisiert und strukturiert. In Anlehnung an die Frame-Theorie der Künstlichen-IntelligenzForschung werden die Strukturkonstituenten eines Frames als Leerstellen, Füllwerte und Standardwerte bezeichnet. Leerstellen werden in der konkreten „Verwendung“ des Frames (etwa beim Verstehen sprachlicher Ausdrücke) mit Füllwerten ausgefüllt. Fungieren bestimmte Werte häufig als Füllwerte der Leerstellen, verfestigen sich diese kognitiv (also im Frame selbst) und werden zu Standardwerten. Die Konzeption der Standardwerte trägt den impliziten Voraussetzungen und Annahmen, die wir beim sprachlichen Austausch machen, Rechnung, weshalb sie bezogen auf die Untersuchung eines Diskursausschnittes von großem Nutzen sind. Zudem ist die Perspektivierung von Sachverhalten als Funktion von Frames in der vorliegenden Untersuchung von großer Bedeutung.

P. A. Neumair, Die Bedeutung von Flüchtling, Geflüchtete_r und Migrant_in, 


\subsection{Zur Entstehung und theoretischen Fundierung der Frame-Semantik}

Die Frame-Semantik stellt einen theoretischen und methodischen Rahmen zur kognitionslinguistischen Bedeutungsanalyse dar. Sie setzt sich zum Ziel, die Bedeutung sprachlicher Äußerungen (Wörter, Phrasen, Sätze, Texte) zu erfassen, indem sie der Frage nachgeht, welches Wissen Rezipient_innen repräsentationell zur Verfügung haben (müssen), um eine solche sprachliche Äußerung verstehen und gebrauchen zu können (vgl. Ziem 2009: 212). Nach der linguistischen FrameTheorie sind sprachliche Äußerungen also keine Bedeutungsträgerinnen, sondern evozieren Bedeutung in Form verstehensrelevanten Wissens. Einige Grundideen der Frame-Theorie, die sich in verschiedenen wissenschaftlichen Disziplinen (Soziologie, Künstliche-Intelligenz-Forschung, Kognitionspsychologie, Linguistik u. a.) unter stärkerer oder schwächerer gegenseitiger Beeinflussung entwickelte, haben ihren Ursprung in der Gestaltpsychologie: So herrscht dort Konsens darüber, dass Objekte der perzeptuellen Wahrnehmung einen gestalthaften Charakter aufweisen und auf der Basis allgemeiner psychologischer Prinzipien konstruiert werden. Ein solches Prinzip lautet: Eine Gestalt ist mehr als die Summe ihrer Einzelteile (ders.: 209). Ebenso einflussreich war die Gedächtnistheorie Frederic C. Bartletts (ders.: 210; Busse 2012: 311 ff.), der entgegen dem seinerzeit vorherrschenden behavioristischen Paradigma den Begriff des Schemas formulierte: "Schema" refers to an active organisation of past reactions, or of past experiences, which must always be supposed to be operating in any well-adapted organic response" (Bartlett 1932: 201). Das Konzept der Schemata war und ist so vage formuliert es bisweilen auch ist und so oft nach dem jeweiligen Interesse der Forschenden zurechtgelegt wurde (Ziem 2008: 266) - für die Entwicklung der linguistischen Frame-Theorie maßgeblich (Ziem 2009: 209). Ziem (2008: 272) kommt gar zu dem Ergebnis, dass sich Frames strukturell und funktionell kaum von Schemata unterscheiden. ${ }^{1}$

Charles Fillmore (1982 u. a.) führte den Frame-Begriff angelehnt an die Gestalt- und Gedächtnispsychologie in die Linguistik ein. Das linguistische Frame-Konzept hatte zu Beginn jedoch noch nichts mit einem kognitiven Deutungsakt gemeinsam: Syntaktische Frames wurden als Kontexte verstanden, die

\footnotetext{
${ }^{1}$ Auf eine ausführliche Auseinandersetzung mit dem Schema-Begriff wird an dieser Stelle verzichtet. Es sei auf die im Text angegebenen Zitationen sowie auf Scheufele 2003 verwiesen, der den Frame- und Schema-Begriff aus kommunikationswissenschaftlicher Sicht behandelt.
} 
, slots ' definierten und Kriterien für die Mitgliedschaft in einer bestimmten Wortklasse bereitstellten; so kann der Frame THE VERY _ THING im Englischen einen Kontext für pränominale Adjektive repräsentieren (Fillmore 2006: 615). Diese enge, syntaktische Lesart von Frame erweiterte Fillmore in seiner Kasusgrammatik (1968): Er führte den Terminus case frame (Kasusrahmen) ein, um nun insbesondere die semantische Valenz von Verben zu beschreiben. Die semantische Valenz eines Verbs sollte unabhängig von der grammatikalischen Realisierung (d. h. Oberflächenstruktur) der Verbargumente identifiziert werden können. So werden zwar im Satz „Tina kauft ein Buch“ lediglich die Tiefenkasus Agentive (in Tina) und Objective (in ein Buch) sprachlich realisiert, die Leser_innen aktivieren jedoch weiteres verstehensrelevantes (Busse 2012 u. a.) Wissen, etwa dass es auch eine_n Verkäufer_in gibt, mit dem/der Tina als Käuferin Geld austauscht, oder dass Tina das Buch im Internet oder in einer Bibliothek erwirbt. Erklärt werden kann dies dadurch, dass beim Lesen des Satzes ein kognitives Modell aktiviert wird, das man mit HANDEL_KAUFEN benennen kann. Den Kern dieses Frames bilden die Frame-Elemente „Käufer“ (Tina) und „Waren“ (ein Buch), während „Geld“ und „Ort“" periphere Elemente sind. ${ }^{2}$ Im maßgeblich von Fillmore initiierten Projekt FrameNet (vgl. Baker et al. 1998) und dem auf die deutsche Gegenwartssprache ausgerichteten Pendant FrameNet des Deutschen (https://gsw.phil.hhu.de/framenet/) werden zahlreiche solcher semantischen Frames sowie lexikalische Einheiten, die diese Frames evozieren, gesammelt. ${ }^{3}$ Ein Frame ist nun nach Fillmore (1982: 111) zu verstehen als ein System von Konzepten, die untereinander derart aufeinander bezogen sind, dass man, um eines dieser Konzepte zu verstehen, die gesamte Struktur, in die dieses Konzept passt, verstehen muss. Wenn also eines dieser Elemente in einer solchen Struktur in einen Text oder in ein Gespräch eingeführt wird, werden automatisch alle anderen kognitiv verfügbar gemacht (Ebd.).

Die Frame-Theorie ist keineswegs eine homogene Theorie, sondern besteht aus unterschiedlichen, mehr oder minder kompatiblen Herangehensweisen. Dies

\footnotetext{
2 https://gsw.phil.hhu.de/framenetold/frame?id=349 (06.12.2021).

${ }^{3}$ Den Unterschied zwischen den Kasusrahmen der Kasusgrammatik und den Frames der Frame-Semantik beschreibt Fillmore (2006: 616) retrospektiv so: „The case frames started out as clusters of participant roles using, initially, names from an assumed universally valid finite inventory of such roles and it was thought that any verbal meaning could be seen as using some collection of these. The frames of current frame semantics, in contrast, are described in terms of characteristics of the situation types themselves, including whatever could be said about the background and other associations of such situations. Moreover, the goal of limiting the number of semantic roles to a roster of reusable universal role names lost its importance, and the effort to force any frame-specific semantic role into the members of such a list could be abandoned in good conscience."
} 
überrascht kaum, da Frame-Konzepte in zahlreichen, unterschiedlichen Disziplinen verwendet werden. In jüngerer Zeit sind jedoch Bestrebungen, die verschiedenen Disziplinen (und damit die jeweiligen Frame-Konzeptionen) in einen Dialog treten zu lassen, zu erkennen (vgl. den 2018 von Ziem/Inderelst/Wulf veröffentlichten Sammelband „Frames interdisziplinär"). Doch auch innerhalb der Linguistik sind Unterschiede hinsichtlich theoretischer Prämissen und methodischer Verfahren festzustellen. Was die methodische Herangehensweise betrifft, sind neben dem Frame-Ansatz von Fillmore und dem Berkeley FrameNet bzw. FrameNet des Deutschen insbesondere die auf die Frame-Konzeption des Kognitionspsychologen Lawrence Barsalou (1992) zurückgehenden „Barsalou-Frames“ zu nennen, für die vor allem im Bereich der Formalen Semantik plädiert wird (vgl. Löbner 2018). Einer der wichtigsten Unterschiede ist, dass in FrameNet nicht von Leerstellen (oder Attributen) und Standardwerten, sondern von FrameElementen gesprochen wird. Wir orientieren uns in der vorliegenden Arbeit zum einen an der Frame-Konzeption des Künstliche-Intelligenz-Forschers Marvin Minsky (1974), indem wir von Leerstellen, Füllwerten und Standardwerten sprechen, zum anderen aber auch an Barsalous Frame-Konzeption, die auf nominale Lexeme zielt. Zugleich ist durch den Umstand, dass sämtlichen zu untersuchenden Ausdrücken Verben zugrunde liegen, eine Zuhilfenahme der Valenzstellen bzw. semantischen Rollen dieser Verben bei der Frame-Analyse sinnvoll, wodurch auch die Fillmoresche Frame-Konzeption Eingang findet (s. o.).

Ohne sämtliche Unterschiede in ihrem vollen Umfang darstellen zu können, sei an dieser Stelle noch der Gegensatz holistischer und modularistischer Positionen in der germanistisch-linguistischen Frame-Semantik genannt. Das eine „Lager“ der Frame-Semantiker (wozu Busse (2012 u. a.) und Ziem (2008 u. a.) zählen) verficht eine holistische Auffassung von Bedeutung und Kognition, die sich entgegen der These der Modularität des Geistes (Fodor 1983) positioniert und auch der vorliegenden Arbeit zugrunde liegt. In einem holistischen Kognitionsmodell gilt:

Sprache, Perzeption und Motorik inferieren und interagieren miteinander. Sie bilden keine autonomen Kenntnissysteme mit eigenen Verarbeitungsprinzipien, sondern sind Teil eines kognitiven Verarbeitungskreislaufes, der nach außen, d.h. zur dinglichen Umwelt, offen ist (Ziem 2008: 112).

Es gibt also grundlegende kognitive Fähigkeiten, die für sämtliche Wahrnehmungsmodalitäten gelten (Ziem 2005: 4). Sprachliche Fähigkeiten hängen stark mit generellen Denkfähigkeiten zusammen (Ziem 2008: 103) und uniforme kognitive Operationen leiten das Verstehen von Sprache (neben weiteren kognitiven Leistungen) (ders.: 106). Dieser holistische Ansatz bezieht sich nicht nur auf 
die Gesamtheit der menschlichen Kognition, sondern auch auf deren Teilkomponenten, wie die Sprache: Im Gegensatz zu am Generativismus und Nativismus orientierten Auffassungen wird eine strikte Trennung von Lexikon, Semantik und Syntax entschieden verneint. Das bedeutet bezogen auf eine Frame-Semantik: Es wird verneint, dass den ,kontextabhängigen Gebrauchsvarianzen eines Ausdrucks ein grammatisch determiniertes Muster zugrunde liegt" (Ziem 2008: 105), wie es z. B. bei Schwarz (1992 u. a.), die ein gestuftes Semantikmodell vertritt und sich zwischen Holismus und Modularismus verortet, ${ }^{4}$ der Fall ist. Vielmehr heißt es, die „Bedeutungserklärung“ könne allein auf Basis konzeptueller Prozesse vorgenommen werden (Ziem 2008: 105). Selbst hinter vorgeblich ambigen Ausdrücken stünde ein kognitives Modell, ein Frame, der diese Ausdrücke kontextualisiert und eine Disambiguierung leiste (ders.: 105 f.). Es sei hier auch an den Umstand erinnert, dass sprachliche Ausdrücke in aller Regel niemals von einem außersprachlichen und/oder sprachlichen Kontext isoliert auftreten. Diesem Gedanken folgend liegt nahe, dass die Bedeutung einer sprachlichen Äußerung bei jeder semantischen Untersuchung lediglich teilweise, nämlich mittels der Herausarbeitung von Teilbedeutungen, greifbar gemacht werden kann und eben nicht die Bedeutung als Ganzes (vgl. Felder 2006a: 18 f.).

\subsection{Frames und ihre Strukturkonstituenten}

Jede sprachliche Einheit ist von Natur aus semantisch stark unterspezifiziert (Ziem 2008: 24), obgleich diese Unterspezifikation wohl einen wichtigen Aspekt reflektiert: Der geringe Inhalt, der ,bereits da ist“, ist bezüglich der Bedeutung der sprachlichen Einheit gleichsam der kleinste gemeinsame Nenner (in) einer Sprachgemeinschaft. Auf diesen Inhalt konnten und können sich die Angehörigen der Sprachgemeinschaft einigen; er emergierte aus Sprachaushandlungsprozessen. ${ }^{5}$ Was die sprachliche Einheit dann konkret bedeutet, hängt stets vom situativen wie sprachlichen Kontext $a b,{ }^{6}$ jedoch ebenso von den Verstehenden, da jede_r stets unterschiedliche Erfahrungen gemacht hat (auch in dem

\footnotetext{
${ }^{4}$ Nach Ziem (2009: 211) dominierten Modelle dieser Art in der germanistisch-linguistischen Rezeption der Frame-Semantik lange Zeit.

${ }^{5}$ Man denke hier auch an die Arbitrarität der Beziehung von Form und Inhalt. Erst durch Konventionalisierung wird einer Form Bedeutung verliehen.

${ }^{6}$ Vgl. Wittgensteins Mantra „Die Bedeutung eines Wortes ist sein Gebrauch in der Sprache“ (Wittgenstein 1953: §43).
} 
Sinne, dass Interaktant_innen einander gewisse Intentionen zu unterstellen vermögen). So folgert auch Ziem (ebd.): Verstehen setzt erst dann ein, wenn diese Unterspezifikation durch zumindest vorläufige Annahmen aufgehoben wird.

Diese Annahmen bilden die Basis der Standardwerte eines Frames: Dies sind Werte, die stets relativ zu unseren bisherigen Erfahrungen, die wir in der Interaktion mit der Welt gemacht haben, erwartbar sind. Sie haben sich (auf sozialer, überindividueller Ebene) durch Konventionalisierung als auch (auf individueller Ebene) wiederholte Perzeption sowie Integration herausgebildet und im (kollektiven) Gedächtnis verankert. In der Wissensstruktur des Frames sind typische Situationen repräsentativ gespeichert. So stellen sich unterschiedliche Menschen bei dem Wort Apfel unterschiedliche Arten von Äpfeln (Referenzobjekte), insbesondere hinsichtlich der Farben (wie rot oder grün), vor. Derartige Standardannahmen bleiben so lange aufrechterhalten, bis eine Instanz des allgemeinen Frames angetroffen wird. Diese Instanz kann das sprachliche Zeichen in einem bestimmten Text oder auch ein konkreter, haptisch oder visuell wahrnehmbarer Apfel (wie etwa in einem Supermarkt) sein. Diese Begegnung mit der Instanz führt im aktualen Wahrnehmungs- und Verstehensprozess zu einem Verdrängen der Standardwerte des allgemeinen Frames: An deren Stelle treten für den Moment neue Werte, die Füllwerte (vgl. Lönneker 2003: 9), die sich aus der sprachlichen oder außersprachlichen Situation ergeben. Standardwerte sind daher Werte, die bereits besonders häufig als Füllwerte der Leerstellen fungierten und sich dadurch kognitiv verfestigten.

Unseren Erachtens liegt nahe, dass mehrere Standardwerte parallel koexistieren können, wobei eine Zuschreibung von Werten der Kategorie der Standardwerte kontext-spezifisch geschieht. Zur Veranschaulichung ein Beispiel: Im Alltag, in der „realen“ Welt, in der die Menschen sich bewegen, die ihnen haptisch zur Verfügung steht, ist die Leerstelle „Art der Fortbewegung“ des Frames zu Auto mit an Sicherheit grenzender Wahrscheinlichkeit in jedem Fall mit dem Wert „fahren“ besetzt, mehr noch kann dieses Konzept als Standardwert festgehalten werden. Geht man jedoch ins Kino, um einen Science-Fiction-Film anzusehen, wird es kaum überraschen, dass Autos in diesem Film fliegen können. Von einem Überschreiben eines Standardwertes durch einen kontext-bedingten „tatsächlichen“ Wert im Moment der (hier visuellen) Perzeption (sobald im Film das erste Mal ein fliegendes Auto zu sehen ist) kann jedoch strenggenommen nicht die Rede sein, da bereits spätestens nach der Auswahl des Films eine Erwartung und Hintergrundwissen aktiviert sind, die mitunter auf vorherigem Konsum von Filmen dieses Genres fußen (können). $\mathrm{Zu}$ diesem Hintergrundwissen gehört auch, dass in Filmen des Science-Fiction-Genres fliegende Autos nicht 
ungewöhnlich sind, weswegen der Wert „fliegen“ im Kontext eines ScienceFiction-Films als Standardwert gesehen werden kann, was jedoch nicht heißen soll, dass in jedem Science-Fiction-Film Autos zwingend fliegen müssen. Wirklich verworfen würde der Standardwert ,fahren“ dann, wenn man nach der Filmvorstellung das Kino verlässt und Autos nun tatsächlich fliegen sieht; die Erwartbarkeit ist eine andere. Dieses Beispiel zeigt auch, dass Frames keinesfalls auf eine Modalität beschränkt sind, sondern als modalitätsunspezifische Repräsentationsformate gelten (vgl. Ziem 2005: 2). Sprachliches und enzyklopädisches bzw. nicht-sprachliches Wissen (,,von Welt") werden nicht getrennt (Busse 2012: 133; Ziem 2008: 113; Ziem 2009: 212).

Füllwerte vermögen im Laufe der Zeit ihre Stellung als „Exot“ zu verlieren: In den 1950er Jahren wäre es wohl ungewöhnlich gewesen, dass Autos batteriebetrieben fahren können; im Jahre 2021 liegt hier ein konkurrierender, da immer häufiger in die Leerstelle ,eingefüllter", Wert vor. Frames sind somit dynamisch. Nicht nur in der diachronen Perspektive, sondern auch synchron betrachtet: Neue Attribute (so nennt der Kognitionspsychologe Barsalou die Frame-Elemente, die anderweitig häufig Leerstellen genannt werden) können konstruiert werden, um neue Aspekte von Exemplaren zu repräsentieren, wenn diese in neuen Kontexten relevant werden (Barsalou 1992: 34).

Ferner enthält ein Frame nach Barsalou zwei Ebenen, er begreift ihn in AntiThese zu den lediglich eine einzige Ebene aufweisenden Merkmalslisten der „klassischen“ Semantiker als Attribut-Werte-Sets (1992: 25 f.). Es ist bedeutend, nicht nur eine Unterscheidung in unterschiedliche Ebenen innerhalb eines Frames vorzunehmen (Attribut - Werte bzw. Leerstellen - Füllwerte), sondern auch Unterscheidungen von Frame zu Frame: Barsalous Begriff von Frames bezieht sich in erster Linie auf Konzept- bzw. nominale Frames, im Kontrast zu Fillmores in der Regel prädikativen Frames. Zudem meint ein allgemeiner Frame eine abstrakte Datenstruktur, die beim Prozess der Identifizierung von Entitäten (als Entitäten eines bestimmten Typs) angewendet wird, ein Muster, das den Verstehenden Orientierung gibt (vgl. Busse 2012: 589).

Frequente Kookkurrenz von Leerstellen in zahlreichen instantiierten ExemplarFrames, d. h. Frames im Kontext, wie sie Barsalou konzipiert, können den Frame-Kern im Allgemeinen bzw. den Kern des Muster-Frames ausbilden. Diese Gruppe von Leerstellen kann dann i.S.v. Prototypikalität als frame-definierend bezeichnet werden. Bezüglich der Leerstellen ist somit eine PrototypikalitätsHierarchie festzuhalten. Damit ergibt sich eine weitere Strukturebene innerhalb eines Frames (Busse 2012: 592). Mit anderen Worten: Manche wie auch immer gearteten Leerstellen werden zu einer Gruppe zusammengefasst, liegen im Zentrum des Frames und sind ,wichtiger“ oder „unabdingbarer“ als andere, in der 
Peripherie liegende Leerstellen. Sie bilden die saliente(re)n Frame-Elemente im Zuge einer Frame-Aktivierung.

\subsection{Die type-token-Problematik und ihre Rolle in der Frame-Semantik}

Für die linguistische Beschreibung der Bedeutung einer lexikalischen Einheit, eines Ausdrucks, muss spezifiziert werden, ob der Gegenstand der Beschreibung eine abstrakte Entität ,in einem gedachten System von Entitäten oder Regeln oder Mustern“ (Busse 2012: 614) einer Gemeinschaft ist oder ein konkretes Auftreten dieser Einheit in einer spezifischen Situation, einem konkreten sprachlichen und außer-sprachlichen Kontext, im Sinne einer Anwendung des Musters (ebd.). Fehlt das Bewusstsein über diese notwendige theoretische wie methodische Differenzierung, ergibt sich nach Busse (2012: 613 f.) die sog. type-token- oder Muster-Exemplar-Problematik, die auch für die angewandte Frame-Forschung von hoher Bedeutung ist. Die type-token-Differenzierung soll an einem Beispiel illustriert werden: Begreift man das Lexem Flüchtling als type, stehen diesem zahlreiche tokens bzw. Anwendungen des Musters gegenüber, etwa die Ausdrücke syrischer Flüchtling oder eritreischer Flüchtling. Diese beiden sind jedoch im eigentlichen Sinne gar keine Exemplare, sondern lediglich Unterkategorien (Exemplar-Typen) für die „echten“ (Einzel-) Exemplare, wie sie in der Welt zu finden sind, die Menschen an sich. Sie dienen also wiederum als Muster bzw. types für die ,in der Welt“ vorkommenden / identifizierten / perzipierten Personen bzw. deren Konzeptualisierung durch die Sprachteilhaber_innen. Es handelt sich hier folglich, wie auch Busse betont (2012: 617), stets um eine relative Zuordnung: Das Dasein einer Entität als type oder token sollte immer in Relation zur nächsthöheren oder nächsttieferen Entität betrachtet werden: Flüchtling ist in Relation zu Ausländer ein token, in Relation zu syrischer Flüchtling oder auch seiner Einbettung in einen konkreten (sprachlichen) Kontext ein type. Dieser Umstand spielt auch in der Frame-Semantik eine wichtige Rolle, denn eine der zentralen Eigenschaften von Frames ist ihre Rekursivität: Die Konzepte, die die Leerstellen von (instantiierten) Frames füllen (Füllwerte), sind häufig selbst als Frames organisiert.

Ein nominaler Konzept-Frame entspricht einem (epistemologischen/kognitiven $)^{7}$ Kategorien-Frame und weist zusätzlich sprachsystematische

\footnotetext{
${ }^{7}$ Busse (2012: 618) führt eine Unterscheidung zwischen einem epistemologischen und einem kognitiven Blickwinkel auf Frames ein. Es wird aber nicht ersichtlich, (a) warum
} 
Aspekte auf (Ders.: 619). (Solche Aspekte werden für die zur Untersuchung stehenden Ausdrücke in Kap. 3 ausführlich behandelt.) Die in dieser Arbeit $\mathrm{zu}$ analysierenden Frames bilden solche nominalen Konzept-Frames in einem spezifischen Kontext, also instantiierte Frames. Diese können Exemplar- bzw. token-Frames genannt werden, wenn Exemplar wie bei Barsalou schlicht als eine höhere, konkretere Stufe in einer konzeptuellen Hierarchie verstanden, ergo relativ betrachtet, wird (s. o.). Falls unter token eine konkrete, außer-kognitive Entität ,in der Welt“ verstanden wird, ist eher von Exemplar-Typ-Frames zu sprechen. Auf der Basis der Ergebnisse soll daraufhin ein allgemeiner, ein type-Frame aufgestellt werden, der sich von einem instantiierten Frame dadurch unterscheidet, dass seine Leerstellen nicht jeweils mehrere unterschiedliche Füllwerte zugleich aufweisen können, sondern durch Standardwerte besetzt werden. Der type-Frame fasst die Bedeutung des jeweiligen Konzepts im untersuchten Diskursausschnitt zusammen (s. 4.4.5). Diskursanalyse wird in der vorliegenden Arbeit ergo als Frame-Analyse in drei Schritten verstanden: Zunächst die Elaboration der Leerstellen der instantiierten Frames, anschließend die Explizierung einer Teilmenge der Gesamtmenge an Füllwerten und zuletzt die Herausarbeitung derjenigen Frame-Elemente, die die jeweiligen type-Frames zu bündeln vermögen. ${ }^{8}$

Eine - neben der Frequenz von Kookkurrenzen - weitere mögliche Unterscheidung von Gruppen von Leerstellen ist eine solche nach inhaltlichem und/oder typologischem Gesichtspunkt, wonach die Meta-Frame-Elemente eine solche Gruppe bilden könnten (Busse 2012: 592): Frame-Elemente mit MetaInformationen betreffen Informationen, die andere Informationen des Frames in bestimmter Hinsicht prägen oder modifizieren, wie Sprecher_inneneinstellungen zum Inhalt der sprachlichen Äußerung (ders.: 586). Semantische bzw. epistemische Frames (d. h. man geht davon aus, dass ein Frame eine Aggregation an Wissen, ein Wissensnetzwerk repräsentiert) integrieren Informationen, die über die üblichen Frame-Elemente, die dinglichen Merkmale, wie sie in der Komponentenanalyse und der Merkmal-Semantik von Bedeutung sind, deutlich hinausgehen (ebd.). Solche Informationen sind Intentionen, Interessen und Einstellungen, Busse nennt diese „spezifische Formen von Eigenschafts-FrameElementen“ (ebd.). Busses Ansatz, derartige Überlegungen in eine Frame-Theorie

diese Unterscheidung notwendig ist und (b) worin der Unterschied der beiden Perspektiven konkret liegt, weshalb in dieser Arbeit nicht weiter darauf eingegangen wird.

${ }^{8}$ Oder anders formuliert: Die Nomina werden in ihrer konkreten syntaktischen Verwendung ,in actu“ analysiert, um so ihrer Bedeutung „de potentia“, als lexikalische Einheit, nahezukommen (vgl. Mumm 1995: 2). 
miteinzubeziehen und gar bei der Konzipierung eines Frames eine zusätzliche Leerstelle für „Bewertung des Referenzobjekts durch die Sprecher“ sowie „Einstellung der Sprecher zum Referenzobjekt“ (jeweils mit den möglichen Werten positiv, negativ sowie graduellen Abstufungen) zu berücksichtigen, damit eine Frame-Beschreibung überhaupt epistemologisch vollständig ist (ders.: 584), ist im Kontext der vorliegenden Arbeit von hoher Bedeutung - bilden öffentlicher und politischer Sprachgebrauch doch ihren Untersuchungsgegenstand.

Die Handlungen der Perspektivierung und der Fokussierung bestimmter Aspekte eines Muster-Frames dienen als Funktionen aktualisierter oder: instantiierter Exemplar(typ)-Frames. Diese Handlungen sind durch Ziele, Interessen und Intentionen gesteuert. Sie geschehen auf zwei Arten: Zum einen als Aktivierung spezifischer (Sub-Sets oder eben „Klassen“ von) Leerstellen aus der Gesamtmenge der Leerstellen eines Muster-Frames, zum anderen als Füllung der Leerstellen mit spezifischen Werten (Busse 2012: 622). Nach Busse sei nun ersteres ,typischer für die Fokussierung bei Frames, die sich auf Objekte mit perzeptueller Überprüfbarkeit ihrer Eigenschaften (sog. Konkreta) beziehen“, während letzteres „typischer [ist] für die Fokussierung bei Frames, die sich auf Objekte ohne perzeptuelle Überprüfbarkeit ihrer Eigenschaften (sog. Abstrakta) beziehen“ (ebd.; Hervorhebung im Original). ${ }^{9}$ Problematisch ist hier, dass, obgleich es gewiss Lexeme gibt, die eindeutig einer dieser Klassen zuzuordnen sind, einige Ausdrücke, wie Flüchtling, Geflüchtete_r und Migrant_in, weder dezidiert als Abstraktum, noch dezidiert als Konkretum bezeichnet werden können - unter der Annahme, dass diese Kategorien nach dem Kriterium der perzeptuellen Überprüfbarkeit gebildet werden. Eine solche dichotomische Unterscheidung ist vielmehr zu verwerfen und eine graduelle, feinkörnige(re) Abstufung vorzunehmen. ${ }^{10}$ Dies lässt sich an den - aus „traditioneller Sicht“ dezidierten Konkreta Tasse und Fußballspielerin illustrieren. Obwohl beide Referenzentitäten ,physische Objekte“ darstellen, ist die Überprüfbarkeit durchaus nicht dieselbe. Deshalb stellt sich die Frage, welcher der oben erläuterten Mechanismen der Perspektivierungs- und Fokussierungshandlungen bei Konzepten wirken, die zwischen Abstrakta und Konkreta stehen, obgleich dies nicht meint, dass sie nicht näher an dem einen als an dem anderen stehen können. Hilfreich ist hier, Abstraktum und Konkretum nicht als Extrempunkte einer Skala zu verstehen, die zugleich einen Gegensatz bilden, sondern „eher [als] Grenzpunkte

\footnotetext{
${ }^{9}$ Die angewandte Frame-Forschung hat bei ihren Untersuchungen bisher jedoch Abstrakta scheinbar gekonnt umschifft.

${ }^{10} \mathrm{Ob}$ der Feststellung in Anm. 9 ist es jedoch nicht verwunderlich, dass es in der FrameSemantik bis dato keine Überlegungen darüber gab, wie eine solche feinkörnige(re) Abstufung aussehen kann.
} 
einer ,Dimension', die sich zwischen realitätsnahen und realitätsferneren, in höherem Grade gedanklich bestimmten Wortprägungen spannt [...]“ (Hempel 1957: 144), wobei „realitätsnah“ und „realitätsfern“ hier wohl so verstanden werden muss, dass eine solche Referenzentität eines sprachlichen Zeichens „realitätsnah" wäre, die sich ,,in der Welt" befindet und unmittelbar perzipierbar ist. Aus einer kognitiv-linguistischen Perspektive mag die Existenz von Konkreta strenggenommen bezweifelt werden, denn Ausdrücke wie Katze oder Haus referieren zwar durchaus auf haptisch wahrnehmbare Entitäten, weswegen sie zunächst den Konkreta zugeordnet würden, bezeichnen jedoch zugleich eine ganze Art von Objekten (type) und nicht die konkreten Objekte an sich (token). Dies geschieht erst durch Kontextualisierung. Falls als Kriterium für eine Zuordnung eines sprachlichen Ausdrucks zur Klasse der Konkreta und der Abstrakta jedoch festgehalten wird, dass bei ersteren potenziell (z. B.) eine (physische) Handlung ausgeführt werden kann, die das Referenzobjekt (in irgendeinem Maße) affiziert (eine Katze kann man streicheln, die Linguistik nicht bzw. nur im metaphorischen Sinn), und man sich zusätzlich der relationalen Bedeutung von type und token bewusst ist, kann eine Unterscheidung in Konkreta und Abstrakta sinnvoll sein. Die type-token-Problematik ist eng mit der Frage nach der Konkretheit bzw. Abstraktheit sprachlicher Zeichen verbunden. Es gibt „,in der Welt“, ,außerkognitiv“, weder den Ausländer noch den Migranten noch den Flüchtling (und auch nicht den Ausländer, den Migranten oder den Flüchtling). Als kognitive Konstrukte, die dergleichen Referenzen im Diskurs ja immer nur sein können, existieren sie jedoch.

Eine strikte Unterscheidung bezüglich sprachlicher Zeichen zwischen Konkretum und Abstraktum kann immer nur vorläufig hilfreich sein. Das, was - wie oben erläutert - Busse für Frames postuliert, nämlich eine graduelle Abstufung (Muster, Exemplar-Typ, konkretes Exemplar), muss nach diesen Erläuterungen im Rahmen einer Frame-Semantik gleichermaßen für die „Verantwortlichen“ der Frame-Evokation gelten - die verwendeten sprachlichen Zeichen. Erst dann kann der Frage nachgegangen werden, welche Mechanismen der Perspektivierung und der Fokussierung konkret wirken.

\subsection{Frames und konzeptuelle Integration (,blending')}

Wie an diesen Mechanismen verdeutlicht, sind Frames sonach mitunter ideologiegeleitet, ideologisch selektiv. Sie werden, wenn sie in der Politiksprache auftreten bzw. eingesetzt werden, zuweilen ,politische Frames“ (Wehling/Lakoff 2016: 67) genannt. Sprachliche Zeichen, d. h. die ausdrucksseitigen, oberflächigen Formen, 
dienen als Anzeichen dafür, dass die Sprachverwender_innen, die das sprachliche Zeichen als Medium der Kommunikation (zwischen ihrem Geist und dem der Adressat_innen) einsetzen, spezifisches Wissen kognitiv aktiviert haben und intendieren, dass auch die Adressat_innen dieses aktivieren (Busse 2012: 537). Deshalb kann es bspw. aufschlussreich sein, Reden politischer Amtsträger_innen $\mathrm{zu}$ untersuchen, bezüglich derer a priori ein intentionales, ideologiegeprägtes Einbetten sprachlicher Zeichen und folglich von Frames in einen spezifischen sprachlichen und kognitiven Kontext festzustellen ist (s. Kap. 4.3). Perspektivierte und fokussierte Frame-Elemente (seien es Leerstellen, seien es Füllwerte) vermögen sich kognitiv zu verfestigen und die Entstehung neuer Muster-Frames zu verursachen, die dann wiederum neue Erwartungen erzeugen. Wird z. B. das Wort Islam von Sprecher_innen wiederholt mit dem Wort Terror in einen Kontext gestellt und von Verstehenden in diesem Kontext perzipiert, „erobern“ Konzepte, die die Leerstellen des Terror-Frames füllen, den Islam-Frame und füllen dessen Leerstellen, wodurch ein neuer Islam-Frame entsteht.

Auf die Theorie der mentalen Räume (,mental spaces'), die im Akt des Verstehens entstehen und durch Frames strukturiert werden (Fauconnier/Turner 1998: 6), angewandt, kann der ,ursprüngliche“ Islam-Frame als ein ,input space“ und der Terror-Frame als ein anderer ,input space" angesehen werden. Zudem sind die beiden Inputs jeweils mit ein und demselben generischen konzeptuellen Raum (,generic space') verbunden; dieser enthält all die strukturellen Elemente, die diese beiden Inputs gemeinsam haben (dies.: 8), im vorliegenden Fall möglicherweise einige Leerstellen. Die ,input spaces' projizieren auf einen weiteren, einen neuen Raum, den ,blend', in unserem Beispiel den neuen Islam-Frame. Die Projektion von Struktur, d. h. spezifischer Frame-Elemente, ist dabei selektiv. Der ,blend“ enthält daraufhin eine emergente Struktur (dies.: 12), die sich in der Art (d. h., dass genau diese Leerstellen mit diesen Werten gefüllt sind) nicht in den Inputs befindet. Diese Anwendung auf Prozesse der konzeptuellen Integration ist jedoch nur sinnvoll, wenn der neue, veränderte Muster-Frame als neue konzeptuelle Einheit begriffen wird.

Im Folgenden soll illustriert werden, wie der oben beschriebene Prozess konkret funktionieren kann: Orientiert am Frame TERRORISM, der dem Berkeley FrameNet nach durch die lexikalische Einheit terror evoziert wird, ${ }^{11}$ können für den Terror-Frame in unserem Beispiel als Leerstellen festgehalten werden: (a) die Aktant_innen, also die Personen, die die Handlung(en) (Terror oder terroristische Akte) ausführen (in der Terminologie semantischer Rollen Agentia)

\footnotetext{
$11<$ https://framenet2.icsi.berkeley.edu/fnReports/data/frameIndex.xml?frame=Terrorism > (06.12.2021).
} 
(entsprechend dem Frame-Element ,Terrorist' im FrameNet), (b) das von diesen Handlungen affizierte Objekt: die Personen(gruppe), gegen die die Handlungen gerichtet sind, als Füllwerte mitunter Hochabstrakta wie die westliche Welt (Patientia) (entsprechend dem Frame-Element ,Opfer'), (c) der Zweck oder ,causa finalis', zu dem die Handlungen ausgeführt werden: im vorliegenden Beispiel enorm ideologiegeprägt (im FrameNet ebenfalls ,Zweck') und (d) die Mittel (,,quibus auxiliis‘?"), die den Aktant_innen gegen die Personen(gruppe) zur Erfüllung des Zwecks oder angestrebten Ziels dienen (im FrameNet ebenfalls ,Mittel').

Zur Feststellung der Leerstellen des Islam-Frame liegt es nahe, einige FrameElemente des Frames RELIGIOUS_BELIEF ${ }^{12}$ zu ,importieren“. Als Leerstellen lassen sich identifizieren: (a) die Angehörigen/Praktizierenden (Agentia) dieser Religion: zusätzlich zu gliedern in Untergruppen (Schiiten, Sunniten u. a.) (entsprechend dem Frame-Element ,Gläubiger' im FrameNet), (b) die konkreten Verhaltensgewohnheiten bzw. die konkrete Ausgestaltung des Glaubens, (c) das Objekt des Glaubens: möglicherweise zu untergliedern in personifizierte und unpersonifizierte Objekte, wobei an letztere auch mitunter Wertvorstellungen gekoppelt sein können (vgl. Ich glaube an Treue) (entsprechend der beiden Frame-Elemente ,Element" und ,Inhalt') und (d) der Zweck oder ,causa finalis ${ }^{6}$ des Glaubens. Die Füllungen der Leerstellen (b) bis (d) sind dabei von der Füllung der Leerstelle (a) abhängig: Unterschiedliche Glaubensrichtungen weisen unterschiedliche Verhaltensweisen auf usw. Leerstellen, die aus den ,input spaces' heraus gemeinsam in den ,generic space' projiziert werden, mögen nun Agentia und Zweck sein. Angehörigen des Islams werden Eigenschaften zugeschrieben, die terroristische Akte ausführenden Personen zugeschrieben werden (wie gewaltverherrlichend, mörderisch), Standardwerte des Terror-Frames treten nun an die Stelle der Füllwerte des Islam-Frames; in diesem Falle gleichermaßen betroffen ist freilich die Leerstelle „Verhaltensgewohnheiten“. Werden für beide Frames zudem die Leerstelle „Einstellung des_der Sprecher_in zum Referenzobjekt" eingeführt, kann ebenso der Füllwert dieser (der bezüglich Terrorismus wohl in aller Regel negativ heißen mag) die Leerstelle des Islam-Frames besetzen. Der ,blended space“ wird alsdann strukturiert durch den neuen Islam-Frame. ${ }^{13}$

\footnotetext{
$12<$ https://framenet2.icsi.berkeley.edu/fnReports/data/frameIndex.xml?frame=Religious_ belief> (06.12.2021).

${ }^{13}$ Der hohen Komplexität der Frames zu Islam und Terror sowie deren konzeptueller Verschmelzung kann im Rahmen dieser Arbeit nicht Rechnung getragen werden. So ist selbstverständlich beispielsweise von einer viel größeren Zahl an Leerstellen bzw. FrameElementen auszugehen.
} 
Die häufige Perzeption und Verarbeitung eines instantiierten Frames in negativem Kontext führt zu einer Veränderung des Muster-Frames bzw. Entstehung eines neuen Muster-Frames, denn

jeder Moment der Frame-Aktivierung ist als Instanziierung einer zunächst abstrakten Struktur [=der type-Frame; PN] mit konkreten Füllungen der mögliche Beginn eines Frame-Umbaus, da Filler/Werte zu Standard-Werten werden können [...] (Busse 2012: 705).

Das führt wiederum dazu, dass, selbst wenn das dazugehörige Lexem in einem neutralen oder sogar positiven Kontext auftritt (unter Umständen auch isoliert), zunächst dieser Muster-Frame aktiviert wird, dessen Leerstellen nun möglicherweise bereits mit negativen Standardwerten besetzt sind. So kann aus kognitiver Sicht ein gewaltverherrlichender, terroristischer Islam zum Prototypen des Islam werden. Es wäre dann „Aufgabe“ des positiven Kontextes, diese negativen Standardwerte durch positive Füllwerte zu ersetzen; ob diese „Aufgabe“ gelingt, hängt dann wiederum von der Robustheit, d. h. vom Grad der kognitiven Verfestigung, der negativen Standardwerte ab. Nun ergibt sich die Frage, ob Leerstellen oder (Standard-)Werte robuster, d. h. widerstandsfähiger, gegenüber neuen Konzepten sind. Dazu sei an dieser Stelle gesagt: Es ist durchaus möglich, dass dies für die Leerstellen gilt, da diese, so wird angenommen (Barsalou 1992: 30), den Frame-Kern bilden. Dieser Frage widmet sich Kap. 4 jedoch noch ausführlicher.

Open Access Dieses Kapitel wird unter der Creative Commons Namensnennung 4.0 International Lizenz (http://creativecommons.org/licenses/by/4.0/deed.de) veröffentlicht, welche die Nutzung, Vervielfältigung, Bearbeitung, Verbreitung und Wiedergabe in jeglichem Medium und Format erlaubt, sofern Sie den/die ursprünglichen Autor(en) und die Quelle ordnungsgemäß nennen, einen Link zur Creative Commons Lizenz beifügen und angeben, ob Änderungen vorgenommen wurden.

Die in diesem Kapitel enthaltenen Bilder und sonstiges Drittmaterial unterliegen ebenfalls der genannten Creative Commons Lizenz, sofern sich aus der Abbildungslegende nichts anderes ergibt. Sofern das betreffende Material nicht unter der genannten Creative Commons Lizenz steht und die betreffende Handlung nicht nach gesetzlichen Vorschriften erlaubt ist, ist für die oben aufgeführten Weiterverwendungen des Materials die Einwilligung des jeweiligen Rechteinhabers einzuholen.

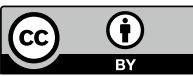

